\title{
La transferencia de conocimiento en las alianzas estratégicas para la innovación ${ }^{1}$
}

\section{The transfer of knowledge in strategic alliances for innovation}

\author{
Manuel Alejandro Díaz Gamarra \\ Pontificia Universidad Católica del Perú \\ manuel.diaz@pucp.pe
}

\begin{abstract}
El presente artículo revisa la literatura sobre alianzas estratégicas para ilustrar cómo son caracterizadas, qué tipos existen y, específicamente, qué fenómenos son relevantes cuando la alianza busca facilitar la innovación empresarial. En este sentido, también se revisa la forma en que la literatura caracteriza los conceptos de innovación, aprendizaje y conocimiento, así como las capacidades organizativas, como factores importantes del proceso de transferencia de conocimiento entre los socios.
\end{abstract}

Palabras clave: alianzas estratégicas, innovación, transferencia de conocimiento

This paper reviews the literature on strategic alliances to illustrate how they are characterized, what types exist and, specifically, what phenomena are relevant when the alliance seeks to facilitate business innovation. In line with this purpose, it also examines the way literature characterizes the concepts of innovation, learning and knowledge, as well as organizational capabilities, as important factors of the process of knowledge transfer between partners.

Key words: strategic alliances, innovation, knowledge transfer

\footnotetext{
1. El presente artículo está basado en una propuesta de investigación anterior —Díaz (2017) — que, sobre la base de una revisión de literatura similar, tiene el objetivo central de proponer una investigación empírica y cuantitativa que no es parte del presente artículo. Dado que ambos trabajos tienen propósitos distintos, pero parten de una línea de investigación común, se prefiere dejar en claro tanto su diferencia como su vinculación.
} 


\section{Introducción}

Cada vez se reconoce más a las alianzas como una herramienta clave para la construcción de ventaja competitiva en las empresas. En los entornos de alta competencia de la actualidad, las alianzas sirven a las organizaciones como un mecanismo para acceder a nuevos insumos del entorno (materiales o intangibles) sin necesidad de desarrollarlos por sí solos (Pittaway, Robertson, Munir, Denyer \& Neely, 2004). El objetivo central del presente trabajo es hacer una revisión de la literatura reciente acerca de cómo son caracterizadas las alianzas estratégicas, qué tipos existen (Faulkner, 2006) y, específicamente, qué fenómenos se identifican como importantes para los procesos de transferencia de conocimiento (Meier, 2011).

Las alianzas son esfuerzos de colaboración de organizaciones que se forman para facilitar la consecución de objetivos estratégicos de los socios (Das \& Teng, 1998). Representan una paradoja de gran interés tanto para académicos como para gerentes (Gomes, Barnes \& Mahmood, 2014) dado que son una estrategia frecuente entre empresas, pero a la vez registran altos porcentajes de fracaso (Dyer, Kale \& Singh, 2001; Park \& Ungson, 2001; Szulanski, 2003; Szulanski, Ringov \& Jensen, 2016; Young-Ybarra \& Wiersema, 1999). Esto hace pertinente explorar cómo caracterizan sus procesos más importantes las investigaciones recientes y el impacto que estas generan en las organizaciones.

La transferencia de conocimiento es un proceso importante en toda alianza, pero se hace especialmente relevante cuando las alianzas tienen como objetivo central dar acceso a nuevo conocimiento a los socios con el objeto de que los ayude en la generación de innovación (Jiménez-Jiménez \& Sanz-Valle, 2011). Dada la creciente importancia de los recursos intangibles como el conocimiento para el sector empresarial (Grant, 2006), así como la necesidad de generar capacidades y procesos para buscarlo activamente en el entorno (Chesbrough, Vanhaverbeke \& West, 2006; Lichtenthaler, 2011), es importante fomentar vínculos de cooperación en diversos sectores para subsanar carencias de conocimiento y estimular el desarrollo de innovación.

El aporte del presente trabajo es presentar la base teórica que sostiene una de las acciones organizacionales más recurrentes en la actualidad, buscando entenderla en términos de su racionalidad; y, más específicamente, cuando se plantea como instrumento para el desarrollo de innovación.

\section{Alianzas estratégicas}

\subsection{Definición de alianzas}

Las alianzas han recibido diferentes definiciones que aportan matices de interés a revisar. Algunos autores resaltan que son relaciones cooperativas que se pueden entender como una adaptación frente a las incertidumbres del entorno (Chen, Gallagher \& Park, 2002). Otros las definen como una relación donde los socios contribuyen a un objetivo común, pero mantienen su independencia (Marshall, Nguyen \& Bryant, 2005; Wang \& Miao, 2006). Gulati y Singh (1998) mencionan que esta colaboración involucra recursos que se «intercambian o codesarrollan», aclarando el tipo de actividad que se desarrolla principalmente. 
Tomaremos esta última definición para decir que las alianzas son «colaboraciones voluntarias entre organizaciones que involucran intercambio de productos, compartir o codesarrollar productos, desarrollo de tecnología o la provisión de servicios con el propósito de perseguir un conjunto de objetivos comunes» (Gulati, 1998). De esta definición se derivan dos elementos importantes: la existencia de participantes (organizaciones) y una relación, que es la materia de interés (Smith, Carroll \& Ashford, 1995; Van Wijk, Jansen \& Lyles, 2008)².

Siguiendo el análisis en estos dos niveles, Todeva y Knoke (2005) presentan tres características importantes de las organizaciones participantes en el contexto de una alianza: que permanecen independientes durante el desarrollo de la alianza, que comparten el control y los beneficios de la alianza, y que finalmente contribuyen con recursos a la relación. Estas características permiten identificar atributos y roles claves que asumen los actores que conforman una alianza; sin embargo, lo más importante es la caracterización de la relación que forman.

Cropper, Ebers, Huxham y Ring (2009), al describir estas relaciones, identifican diversos términos en la literatura que clasifican como nombres o sustantivos para este vínculo, adjetivos que lo caracterizan y verbos que indican cierta funcionalidad (ver tabla 1). Todo ello evidencia una gran cantidad de matices para las alianzas identificados en la literatura; no obstante, todos confluyen en la necesaria existencia de al menos dos organizaciones que la conforman y de una relación colaborativa entre estas.

\section{Tabla 1. Términos asociados a alianzas estratégicas}

\begin{tabular}{l|l|l} 
sustantivos & adjetivos & verbos \\
\hline - Alianza & - Colaborativa & - Conectar \\
- Colaboración & - Interorganizacional & • Franquiciar \\
- Federación & - Multiagente & - Colaborar \\
- Asociación & - Transorganizacional & - Tercerizar \\
- Sociedad & - Cooperativa & \\
- Consorcionada & \\
- Joint venture & Entrelazada & \\
- Relación & & \\
- Clúster & & \\
- Red & & \\
- Coalición & & \\
- Cooperativa & &
\end{tabular}

Fuente: Díaz (2017), adaptado de Cropper et al. (2011).

\subsection{Resultados esperados de las alianzas}

Las alianzas como instrumentos estratégicos buscan impactar positivamente en las organizaciones que las implementan. Una revisión de la literatura reciente clasifica estos impactos como resultados en términos contables, en términos de percepción

2. Otra característica importante de una alianza es que es estable al menos durante un periodo de tiempo delimitado. Por ello, se dice que va más allá de transacciones de mercado puntuales (Geyskens et al., 2006). 
de accionistas, en términos de estabilidad de la alianza y otras medidas subjetivas que consideren relevantes los socios de la colaboración (Christoffersen, Plenborg \& Robson, 2014). Otra forma de clasificar los resultados esperables se relaciona con el "foco" que los socios deciden darle en términos de impacto final (por ejemplo, resultados financieros), proceso (por ejemplo, innovación o aprendizaje) o estructuras claves en las organizaciones (confianza, justicia o relaciones claves). Es importante notar que diferentes enfoques teóricos dan lugar a distintas conceptualizaciones de resultados esperables de las alianzas, como se puede ver en la tabla 2 (Provan \& Sydow, 2009).

Tabla 2. Definiciones de performance de alianzas

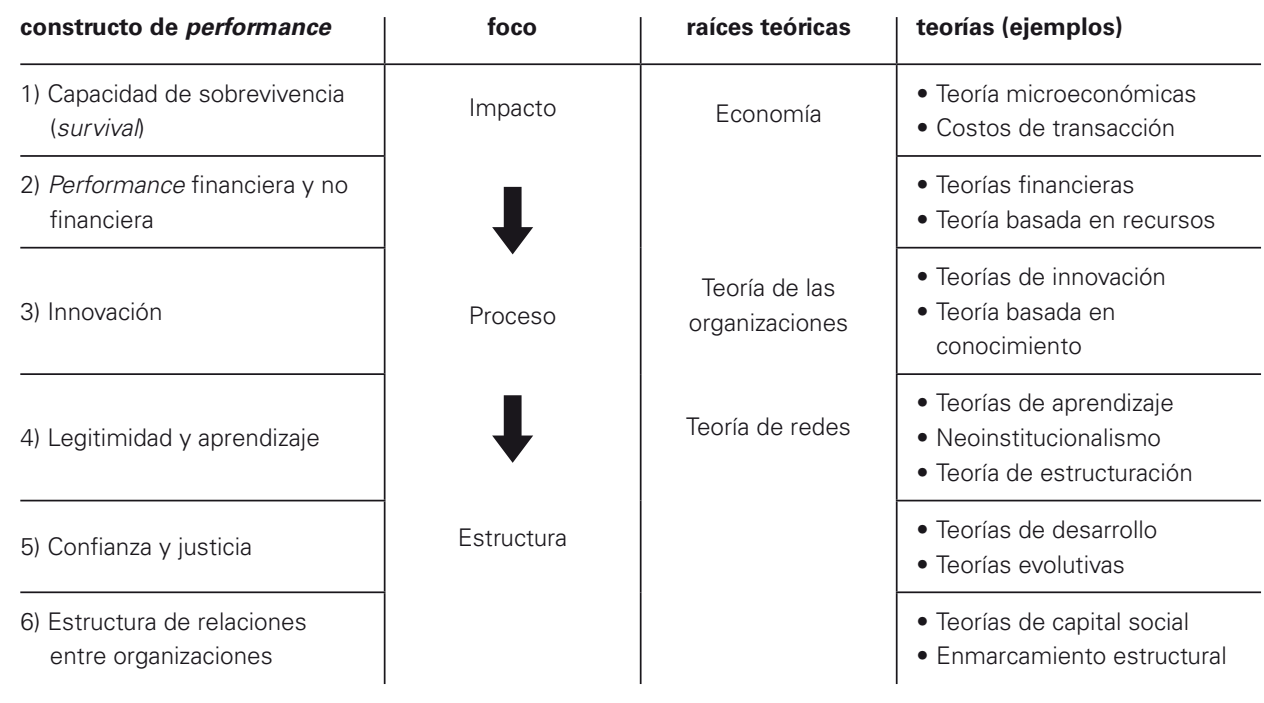

Fuente: Díaz (2017), adaptado de Provan y Sydow (2009)

Las alianzas de interés para el presente trabajo son aquellas que buscan generar innovación. Estas se construyen desde enfoques basados en el aprendizaje y el conocimiento (Inkpen \& Tsang, 2007; Khanna, Gulati \& Nohria, 1998; Kogut, 1988; Kogut \& Zander, 1992). Por ello, es relevante aclarar los principales marcos teóricos que explican la racionalidad de las alianzas.

\subsection{Racionalidad de las alianzas - enfoques adaptativos}

Las alianzas han sido estudiadas desde diferentes teorías, que han resaltado diversos aspectos de su racionalidad. Todas estas perspectivas colaboran a la construcción de un entendimiento amplio de este fenómeno, aun cuando algunas teorías son más vigentes o han ganado mayor atención en la literatura reciente.

Podemos observar que las alianzas son entendidas como respuestas más proactivas en la medida en que vamos acercándonos a perspectivas más contemporáneas. Un primer grupo de teorías (sistemas, contingencias, dependencia de recursos y costos de transacción) entiende a las alianzas como un esfuerzo de búsqueda de información y adaptación frente al entorno, el cual toma la forma de adaptación en la estructura interna 
mediante acciones políticas o la selección de actividades internas en función de optimización de costos. Un segundo grupo de teorías (basado en recursos y en conocimiento) las entiende como formas activas de crear ventajas competitivas. Esta última perspectiva nos permite entender mejor a las alianzas cuando funcionan como herramientas para crear innovación.

Tabla 3. Teorías sustento de alianzas estratégicas

\begin{tabular}{l|l} 
teoría & motivos de la alianza estratégica \\
\hline $\begin{array}{l}\text { Teoría general de sistemas } \\
\text { (Systems theory) }\end{array}$ & $\begin{array}{l}\text { Búsqueda de recursos para adecuación óptima (Johnson \& Rosenzweig, } \\
1964)\end{array}$ \\
\hline $\begin{array}{l}\text { Dependencia de recursos } \\
\text { (Resource dependency) }\end{array}$ & $\begin{array}{l}\text { Adquisición de recursos y disminución de incertidumbre mediante influencia } \\
\text { política (Pfeffer \& Salancik, 1978) }\end{array}$ \\
\hline $\begin{array}{l}\text { De contingencias } \\
\text { (Contingency) }\end{array}$ & $\begin{array}{l}\text { Reducción de incertidumbre mediante adaptación estructural (Ranganathan } \\
\text { \& Lertpittayapoom, 2002) }\end{array}$ \\
\hline $\begin{array}{l}\text { Costos de transacción } \\
\text { (Transaction cost) }\end{array}$ & $\begin{array}{l}\text { Riesgo de ventajas en costos y reducción de incertidumbre (Williamson, } \\
1985)\end{array}$ \\
\hline $\begin{array}{l}\text { Basado en recursos } \\
\text { (Resource based view) }\end{array}$ & $\begin{array}{l}\text { Adquisición de recursos, construcción de ventaja competitiva (Tsang, 1998; } \\
\text { Das \& Teng, 2003; Yasuda, 2005) }\end{array}$ \\
\hline $\begin{array}{l}\text { Basado en conocimientos } \\
\text { (Knowledge-based view) }\end{array}$ & $\begin{array}{l}\text { Adquisición de conocimiento, aprendizaje organizacional, construcción de } \\
\text { ventaja competitiva (Van Gils \& Zwart, 2004) }\end{array}$ \\
\hline $\begin{array}{l}\text { Capacidades dinámicas } \\
\text { (Dynamic capabilities) }\end{array}$ & $\begin{array}{l}\text { Adquisición de conocimiento en la forma de rutinas o habilidades } \\
\text { organizacionales para la construcción de ventaja competitiva (Augier \& } \\
\text { Teece, 2009; Kale \& Singh, 2007) }\end{array}$
\end{tabular}

Fuente: Díaz (2017), adaptado de Sambasivan, Siew-Phaik, Mohamed y Leong (2013).

La teoría general de sistemas resalta la importancia del correcto vínculo de la empresa con su entorno como una clave para asegurar su supervivencia. La preocupación principal se centra en el conocimiento y la adaptación, y por ello una alianza es un esfuerzo de acceso a recursos necesarios para dicha adecuación (Johnson \& Rosenzweig, 1964; Sambasivan et al., 2013).

Este también es el planteamiento de la teoría de contingencias, que sin embargo se interesa más en entender la forma en que finalmente el entorno afecta la configuración estructural o las estrategias de las organizaciones (Sambasivan et al., 2013). Esta teoría busca entender las diferencias entre organizaciones en función del entorno, y por ello ve a las alianzas como un ejemplo de esa adaptación estructural.

Otro teoría que se centra en entender a las alianzas como respuestas del entorno es la teoría de dependencia de recursos (Pfeffer \& Salancik, 1978). Aunque también parte de la idea de la influencia del entorno, resalta un tipo de respuesta particular de la organización pues, desde esta perspectiva, el entorno plantea una realidad política a la que la organización responde con el mismo tipo de estrategias. En esa línea, Pfeffer (1978) menciona que una organización sobrevive en la medida que asegura insumos claves del entorno mediante el ejercicio del poder o la influencia política.

La teoría de costos de transacción (Coase, 1937; Williamson, 2010) también identifica a las alianzas como una forma de adaptación, pero en este caso la adaptación implica elegir "los límites de la empresa» (Kogut, 1988). Desde este enfoque, toda 
organización es un conjunto de actividades coordinadas por mecanismos de jerarquía interna que se contraponen a las actividades realizadas en el mercado abierto, que son libres o gobernadas entre todos los agentes sin estructuras jerárquicas.

Toda organización debe decidir constantemente si realizar actividades bajo la jerarquía interna o realizarlas mediante contratos con agentes de mercado, por ejemplo, a modo de subcontratación. Ambas tienen ventajas y desventajas: la jerarquía interna permite controlar el oportunismo y promover la cooperación entre quienes realizan las actividades; mientras que los mecanismos de mercado permiten buscar al socio más idóneo para una actividad, además de compartir el riesgo con otros actores. Lo óptimo se define en función de la minimización de los costos, considerando que existen tanto costos de transacción para las operaciones externas (costos de búsqueda de contraparte, negociación para evitar el oportunismo, seguimiento del cumplimiento del contrato) como costos de operación internos (costo del personal a cargo, costo del aprendizaje, costos de supervisión, etcétera) que se deben evaluar para decidir qué opción estratégica tomar (Geyskens, Steenkamp \& Kumar, 2006).

La teoría de costos plantea que una alianza es un punto intermedio entre el espacio interno de la empresa y el mercado (ver gráfico 1). Es una respuesta estratégica que permite no internalizar todas las operaciones, ni tampoco manejarlas todas mediante el mercado, lo que podría ser una forma de adaptación óptima (Das \& Teng, 2000; Ortiz-deUrbina-Criado, Montoro-Sánchez \& Mora-Valentín, 2014).

\section{Gráfico 1. Tipo de actividades realizadas por una alianza}

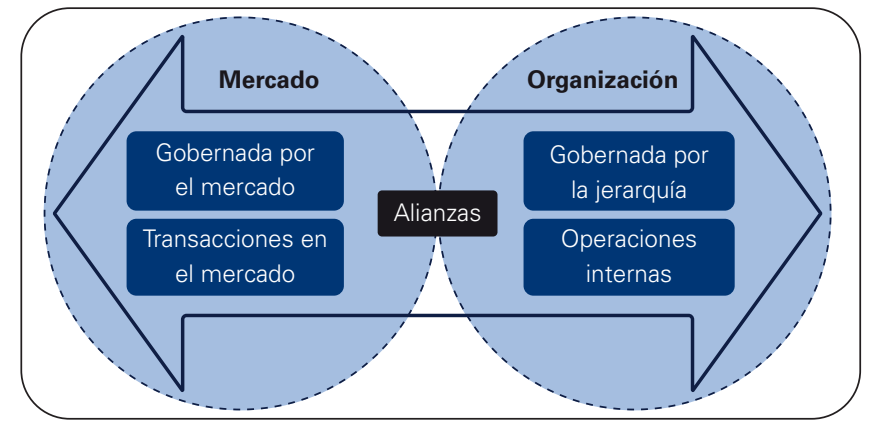

Fuente: Díaz (2017).

\subsection{Racionalidad de las alianzas - enfoques proactivos para la generación de valor}

Las últimas teorías se centran no solo en una adaptación reactiva, sino en una búsqueda proactiva de una mayor capacidad de generar valor. Para la teoría basada en recursos la clave está en adquirir recursos de mayor calidad, puesto que ellos garantizan una mayor capacidad de generar valor tanto para clientes como para la organización (Barney, 1991; Peteraf, 1993). Dado que la competencia hace que las organizaciones busquen y accedan eventualmente a los mismos recursos, muchas veces la calidad también está en función de la escasez de ciertos recursos. Aquellos que no son de fácil acceso en el mercado (escasos, inamovibles e inimitables) serían los más deseados, lo que empujaría a las empresas a acercarse a aquellas organizaciones que los tienen. De esta forma, las alianzas 
serían canales de acceso a recursos escasos bajo una lógica de construcción de ventaja competitiva frente a los rivales (Das \& Teng, 2000; Ortiz-de-Urbina-Criado et al., 2014).

Esta teoría es criticada porque, si bien se considera un razonamiento revelador, deja sin explicar muchos conceptos en los que se basa. No plantea diferencias claras entre recursos y capacidades, además describe la realidad organizacional de forma estática, sin considerar cómo va cambiando en el tiempo la base de recursos en una misma empresa (Augier \& Teece, 2009; Cepeda \& Vera, 2007; Tallman, 2006). Dos teorías posteriores permiten cubrir algunas de estas falencias: el enfoque basado en el conocimiento y la teoría de capacidades dinámicas.

El enfoque basado en conocimiento (Grant, 1996, 2006; Spender \& Grant, 1996) continúa en esa ruta. Se considera una derivación de la teoría basada en recursos, pero se caracteriza por poner al conocimiento como el recurso central en toda organización. Esto implica no solo reconocer que el conocimiento presenta las características de los recursos valiosos (escasos, inamovibles e inimitables), sino que reinventa el sentido mismo de la organización como un procesador de conocimiento. Para ello, esta perspectiva afirma que el conocimiento es el recurso con mayor capacidad de generación de rentas, muy por encima de otros recursos; clasifica el conocimiento como valioso de acuerdo a su dificultad de movimiento (tácito versus explícito, a explicarse después); y entiende la eficiencia organizacional como el aprovechamiento de economías de escala para la búsqueda, recojo, generación y utilización de conocimiento (Grant \& Baden-Fuller, 2004). Estas eficiencias las relaciona también con decisiones de especialización e integración tomadas por las personas dentro de la organización.

Esta perspectiva encuentra complementariedades en teorías anteriores, como la de costos de transacción, para argumentar a favor de una economía basada en intangibles, donde el conocimiento es valioso en función de su mayor velocidad de transporte y menor costo de producción. El costo de otros recursos también tiende a disminuir, pero no en el mismo grado, y ni estos ni otros recursos tienen el mismo nivel de impacto que el conocimiento (Teece, 2010).

Este enfoque incluso diferencia entre las perspectivas de «acceso al conocimiento» y "adquisición de conocimiento». La primera resalta que las empresas están tendiendo asociarse con compañías que tienen un conocimiento que no pretenden absorber, sino únicamente utilizar, en la medida que la alianza permite generar valor para ambos socios sin que sea necesaria la absorción. Esto se explica porque la competitividad requiere que las organizaciones se especialicen en campos específicos, lo que desincentiva incorporar conocimiento nuevo que no agrega profundidad a la especialidad de la organización (Grant \& Baden-Fuller, 2004). Se trata, pues, de un tipo de cooperación que se basa en la sinergia y especialización de los socios, aun cuando en otros casos las organizaciones sí pretenden ampliar su base de conocimiento, como cuando esperan generar innovaciones.

La teoría de capacidades dinámicas conceptualiza con mayor detalle la diferencia entre recursos y habilidades organizacionales. Estas últimas las entiende como rutinas o prácticas aprendidas dentro de la organización que van más allá de los procesos formales para enraizarse fundamentalmente en la dimensión social del colectivo, y las clasifica en un «primer nivel» como habilidades simples y en un "segundo nivel» como habilidades que afectan a otras habilidades. Ese segundo grupo reúne a las capacidades dinámicas, 
que serían las responsables de la generación de una ventaja competitiva sostenible en el tiempo (Barreto, 2010). Sobre estas dos últimas teorías se ampliará más adelante cuando se profundice en torno al conocimiento y el aprendizaje.

\subsection{Tipos de alianzas}

Los estudios previos en alianzas identifican dos formas de categorizarlas. Una primera se enfoca en la estructura que toma la relación, mientras que la segunda se ocupa de la intencionalidad detrás de las alianzas.

Respecto de la estructura (Globerman \& Nielsen, 2007; Gulati \& Singh, 1998; Oxley, 1997), la relación puede implicar la creación de una nueva organización separada de los socios, aun cuando sea dependiente de ellos. Esta estructura propone que el gobierno de la alianza se dé a través de la propiedad de acciones de la nueva entidad y por ello recibe el nombre en inglés de equity partnerships. En oposición, la relación también se puede estructurar únicamente mediante contratos o acuerdos entre las partes, sin crear una nueva organización, lo que recibe el nombre en inglés de non-equity partnerships. La creación de una organización suele representar ventajas en términos de control sobre el oportunismo de las partes, pero requiere un gran nivel de confianza y esfuerzo a comparación de las alianzas llevadas únicamente de forma contractual. También existe evidencia de que la creación de una organización facilita la transferencia de conocimiento complejo o de difícil transferencia; pero, en contrapartida, también suele ser una salida más burocrática y poco ágil en comparación con los mecanismos de colaboración contractuales (Mowery, Oxley \& Silverman, 1996).

Con respecto a la intencionalidad detrás de la colaboración, la literatura divide las alianzas en «de exploración» y «de explotación». Las primeras se centran en la creación de nuevo conocimiento, como sucede al innovar; mientras que las segundas buscan la utilización eficiente del conocimiento ya existente (Koza \& Lewin, 1998; Parmigiani \& Rivera-Santos, 2011). Es importante destacar que, desde la teoría basada en conocimiento, este proceso es inherente a toda organización, independientemente de que se realice internamente o con apoyo externo; es más, justamente se realiza por medio de alianzas cuando es necesario apoyarse en socios que ayuden a implrementarlo mejor (March, 1991).

El caso de la innovación se enmarcaría en esfuerzos de exploración, que se realizan con aliados cuando el conocimiento propio es insuficiente.

\section{Gráfico 2. Tipos de alianzas}

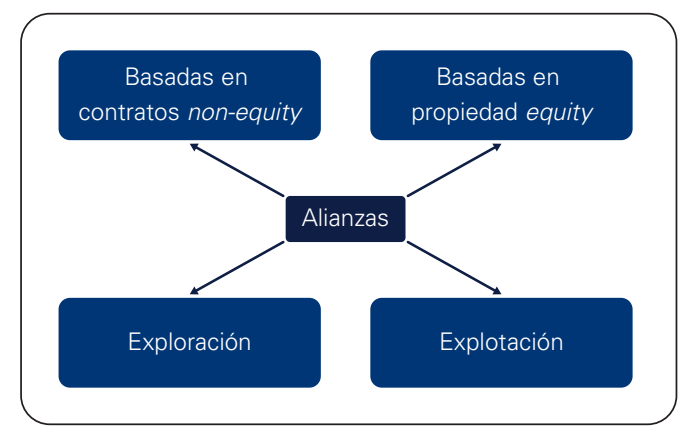

Fuente: Díaz (2017). 


\section{Aprendizaje y transferencia de conocimiento en alianzas para la innovación}

\subsection{Innovación, aprendizaje y conocimiento}

La innovación tiene diferentes definiciones en la literatura, pero en su sentido más simple consiste en la adopción de nuevas ideas o nuevos comportamientos (Jiménez-Jiménez \& Sanz-Valle, 2011) con la intención de mejorar la ejecución de actividades y, por lo tanto, la capacidad de obtener resultados a través de ellas. Específicamente, se ha clasificado a la innovación como mejoras en términos de nuevos productos, nuevas formas de producción o modelos de gestión (Crossan \& Apaydin, 2010).

Es una tendencia de gran interés para las organizaciones, lo que se refleja en un creciente interés a nivel práctico y académico (Gunday, Ulusoy, Kilic \& Alpkan, 2011). Constituye también una importante fuente de ventaja competitiva debido a que, por definición, la innovación es únicamente poseída por quien la produce y, por lo mismo, es escasa en el resto de la industria (Barney, 1991; Helfat \& Peteraf, 2003). Pese a que el cambio y la mejora son inherentes a casi cualquier actividad humana, en el campo organizacional son estudiados e implementados de forma sistemática solo recientemente (Fagerberg, 2004).

Es difícil representar adecuadamente la diversidad de procesos y actividades de innovación que las empresas realizan debido a sus características propias (sectoriales, desarrollo tecnológico, etcétera), y también por los sesgos de los estudios que, al provenir de disciplinas distintas, se centran en aspectos diferentes. Sin embargo, se pueden identificar procesos básicos como la generación de conocimiento, la transformación de conocimiento en herramientas productivas y la capacidad de atender o afectar mejor el mercado (Pavitt, 2009). Lograr el desarrollo de cualquiera de estos procesos sería el resultado esperado por las organizaciones socias de una alianza para la innovación, lo que implica aprendizaje organizacional.

El aprendizaje organizacional es definido como el cambio en las habilidades o rutinas de una organización debido a la interacción entre reflexión y acción organizacional, siguiendo la secuencia de pasos de entender, actuar y luego interpretar/interiorizar (Vera, Crossan \& Apaydin, 2011). La literatura identifica al aprendizaje organizacional como un antecedente importante para la innovación (Jiménez-Jiménez \& Sanz-Valle, 2011) y al conocimiento como insumo central en este proceso (Gray \& Meister, 2004; Tsoukas \& Vladimirou, 2001).

El conocimiento es "la capacidad humana de hacer juicios de valor y distinciones en un campo de acción» (Tsoukas \& Vladimirou, 2001). Esto implica una postura propia basada en la asimilación crítica información de la realidad y la combinación constante de información nueva y antigua como la actividad esencial del aprendizaje (Kogut \& Zander, 1992). Dado que el conocimiento es entendido como una postura propia, es importante mencionar que el conocimiento a nivel de personas se transforma en conocimiento de la organización cuando los juicios de valores se hacen atendiendo a las rutinas y reglas de la organización, las cuales funcionan como una base que estandariza las actividades dentro de cierto rango de respuestas esperables.

Las rutinas organizacionales, sean formales o informales, se entienden además como habilidades de la organización, dado son prácticas que la empresa está 
en la capacidad de realizar (Kogut \& Zander, 1992; Vera et al., 2011). En este contexto, el aprendizaje organizacional consiste en afectar estas capacidades/rutinas organizacionales, lo que podría implicar una ventaja competitiva si se genera una capacidad que el resto de competidores no posee (Barney, 1991; Helfat \& Peteraf, 2003). Considerando la importancia del conocimiento para el aprendizaje y la innovación (Gray \& Meister, 2004), los autores estudian los insumos de conocimiento que provienen tanto de fuentes internas (operaciones propias y aprendizaje interno de la organización) (Smith, Collins \& Clark, 2005; Su, Ahlstrom, Li \& Cheng, 2013) como de fuentes externas (aprendizaje de otras organizaciones o grupos) (Meier, 2011).

Para el segundo caso la literatura de aprendizaje ha coincidido con la predominancia del paradigma de la innovación «abierta» (Chesbrough et al., 2006). Este paradigma consiste en el reconocimiento de que la innovación muchas veces nace de la interacción de las organizaciones con su entorno, y no únicamente de fuentes internas. La innovación abierta no solo consiste en buscar acceso a conocimiento nuevo del entorno, sino que incluso puede incluir la gestión de ese conocimiento fuera de sus límites (Grant \& Baden-Fuller, 2004; Van De Vrande, Lemmens \& Vanhaverbeke, 2006). Utilizada primero solo en ciertas industrias por sus características y necesidades propias (Arora \& Gambardella, 2010), se reconoce que hoy existen cada vez más sectores donde también se utiliza la innovación abierta (Beamish \& Lupton, 2009; Cassiman \& Veugelers, 2006). Incluso hoy las empresas intentan explotar el conocimiento disgregado no solo en uno, sino en diferentes actores a la vez, como clientes, proveedores, universidades, etcétera (Gassmann, 2006). Esto es parte de lo que Chesbrough llamó "aprendizaje inter-organizacional», que es estudiado por teorías de aprendizaje organizacional y de capacidad de absorción (Vanhaverbeke, Van De Vrande \& Chesbrough, 2008); y es lo que hace relevante el proceso de transferencia de conocimiento entre organizaciones, como el que se da en el marco de las alianzas.

\subsection{Transferencia de conocimiento en alianzas y capacidades necesarias para la transferencia}

La transferencia de conocimiento implica la réplica de una parte de la base de conocimiento de una organización, que actúa como fuente, en otra que actúa como receptora. Esto requiere transmisión de información, pero sobre todo un cambio efectivo en las reglas y prácticas formales e informales del conjunto de personas que trabajan en la organización receptora (Tsoukas \& Vladimirou, 2001).

Los beneficios de la transferencia están asociados al aprendizaje de nuevas rutinas e incluso a la generación de nuevas prácticas diferenciadoras, como ya se ha comentado (Lin, Wu, Chang, Wang \& Lee, 2012; Tsai, 2001); no obstante, es importante notar que no solo existen beneficios, sino también costos asociados a los esfuerzos por generar la transferencia de conocimiento en el marco de una alianza. Costos directos como tiempo invertido, tanto en la búsqueda del socio como en la adaptación durante la relación, pueden hacer que el esfuerzo de generar una transferencia sea subóptimo (Levine \& Prietula, 2012). Estudios acerca de los costos de la transferencia de conocimiento en alianzas identifican que en algunos casos, cuando la organización ha invertido fuertemente para tener fuentes de conocimiento a la mano (sistemas de gestión del conocimiento dentro de la empresa 
o mediante el contacto con expertos), los costos pueden sobrepasar los beneficios. Por ello, las inversiones previas de la empresa pueden ser incentivos fuertes para inhibir la colaboración con otras organizaciones. Asimismo, las dificultades o costos de este proceso están asociados principalmente a las características del conocimiento que se desea transferir (Nonaka \& von Krogh, 2009) y a ciertas capacidades necesarias de los socios (Adler \& Kwon, 2002; Van Wijk et al., 2008).

Respecto del conocimiento a transferir, la literatura ha estudiado el concepto de la ambigüedad del conocimiento (Birkinshaw, Nobel \& Ridderstrale, 2002). Esta se refiere a la incertidumbre acerca de cuáles son las dimensiones internas y fuentes subyacentes de un conocimiento, así como cuál es la forma de interacción ideal para su transmisión. La ambigüedad surge de la especificidad y de la complejidad del conocimiento a transferir, y es una barrera importante para su transferencia (Simonin, 1999, 2004). Otra forma de examinar este mismo problema se ha planteado a través del estudio del nivel de preparación para su transferencia de conocimiento tácito y codificado (Inkpen \& Dinur, 1998; Nonaka, 1994; Nonaka \& von Krogh, 2009; Polanyi, 1966; Polanyi \& Sen, 2009). Algunas veces el conocimiento está presente únicamente de forma arraigada en las rutinas de la organización y no se encuentra preparado para su transmisión, lo que se conoce como conocimiento "tácito». Otras veces el conocimiento se encuentra especificado en documentos o está "codificado", lo que implica que está preparado para su transferencia (Grant, 1996; Inkpen \& Dinur, 1998; Meier, 2011). El conocimiento, cuando es tácito, requiere de un esfuerzo especial y de condiciones que no siempre están presentes. Varios estudios muestran, por ejemplo, la necesidad de que la relación permita un acercamiento al contexto del conocimiento requerido, así como lazos fuertes, confianza y valores compartidos en una alianza para lograr la transferencia efectiva de conocimiento tácito o ambiguo (Dhanaraj, Lyles, Steensma \& Tihanyi, 2004).

En el caso de las características organizacionales de los socios, hay muchas variables que pueden afectar la transferencia (tamaño, antigüedad y centralización, entre otras) (Dhanaraj et al., 2004; Frost, Birkinshaw \& Ensign, 2002; Gupta \& Govindarajan, 2000; Laursen \& Salter, 2006; Sheremata, 2000), pero el reto principal se encuentra en tener desarrolladas ciertas "capacidades» de la empresa. La literatura señala que todas las organizaciones tienen capacidades distintas de acuerdo a las rutinas que han desarrollado por sus experiencias pasadas; algunas de ellas son dinámicas, y aquellas son las que permiten aprovechar mejor las alianzas estratégicas (Lin et al., 2012).

Una capacidad organizacional es catalogada como capacidad "dinámica» cuando puede transformar enteramente toda la base de recursos de conocimiento y rutinas de una empresa en función de los requerimientos de un nuevo entorno (Cepeda \& Vera, 2007; Vera et al., 2011). Estas capacidades dinámicas son reconocidas en la literatura como "recursos intangibles complejos» que "dependen de la totalidad del grupo» (Teece, Pisano \& Shuen, 1997) y forman parte de un «tercer nivel» de recursos, el más importante para construir una ventaja competitiva. Las capacidades dinámicas no pueden ser compradas, más bien son desarrolladas en la rutina diaria, de acuerdo a «las demandas operativas específicas de la empresa y una variación aleatoria de los procesos internos» en la empresa (Tallman, 2006). 
Debido a la competencia, eventualmente todas las capacidades no dinámicas son copiadas por empresas competidoras. De esta manera, las empresas se homogeneizan constantemente hasta que alguna vuelve a diferenciarse mediante el desarrollo de cierta capacidad distintiva (aunque temporal), en una lógica de «innovación schumpeteriana» (Chen, Hsiao \& Chu, 2014). En otras palabras, todo el tiempo son nuevas capacidades las que ocupan el lugar preponderante de las capacidades anteriores, siempre que sean lo suficientemente disruptivas e innovadoras. Sin embargo, las capacidades son las responsables de reinventar la empresa y son únicas para cada organización.

Una de las capacidades dinámicas más interesantes es la capacidad de absorción (Cohen \& Levinthal, 1990). Es la habilidad organizacional de «reconocer, asimilar y utilizar» conocimientos nuevos, formada a partir del conocimiento previo de la organización, que funciona "como una base para poder asimilar e incorporar nuevos conocimientos» (Nooteboom, Van Haverbeke, Duysters, Gilsing \& van den Oord, 2007; Noseleit \& de Faria, 2013; Sampson, 2007). Los autores detrás de esta capacidad (Lin et al., 2012) argumentan que las organizaciones requieren invertir primero en desarrollar conocimientos y habilidades básicas para luego aprovechar nuevas oportunidades de aprendizaje del entorno.

Esta capacidad de absorción es identificada por algunos autores como parte de la capacidad de aprendizaje y, al mismo tiempo, como una capacidad dinámica (Vera et al., 2011). Meier ( 2011) indica que esta capacidad no se puede caracterizar de forma aislada a la realidad en la que se encuentra, ya que también se ve afectada por variables como las características del tipo de conocimiento compartido, las características de los socios involucrados en la alianza (Sampson, 2007) y las características de la alianza en sí. Es decir, su mayor o menor efectividad depende de la alianza específica y las características propias de la relación planteada (Lane \& Lubatkin, 1998).

\section{Conclusiones}

La literatura sobre alianzas estratégicas ha crecido de la mano con el incremento de la interacción de organizaciones, así como de las nuevas perspectivas teóricas que buscan entender este crecimiento. La innovación y la innovación abierta, por ejemplo, tendencias muy fuertes en la actualidad, pertenecen a este conjunto de influencias que mueve a las organizaciones a plantear acciones de cooperación interorganizacional.

Existen diversas razones detrás de la cooperación de las organizaciones que van desde perspectivas más adaptativas hasta respuestas más proactivas. Dentro de estas últimas se encuentran las alianzas que funcionan bajo una lógica de innovación, lo cual hace necesario entender los fundamentos del aprendizaje organizacional. El conocimiento implica la reflexión crítica de los datos de la realidad para crear un marco de referencia común dentro del colectivo organizacional. El cambio del marco de referencia y de rutinas es lo que se conoce como aprendizaje organizacional y requiere una transferencia de conocimiento previa que lo facilite.

La transferencia de conocimiento se dificulta en la medida que el conocimiento se hace más ambiguo o tácito, pero esas características están asociadas en la literatura a recursos valiosos para la construcción de ventajas competitivas. Por ello, la transferencia de 
este conocimiento requiere un soporte especial de ciertas habilidades organizacionales o capacidades dinámicas vinculadas al aprendizaje como la capacidad de absorción.

A través de la presente revisión de la literatura se han presentado las bases teóricas que explican la transferencia de conocimiento, el aprendizaje y la innovación. De esta forma, se pretende no solo aportar al entendimiento de las alianzas de forma aislada, sino relacionarlo claramente con el planteamiento de estrategias adaptativas y de creación de ventajas competitivas en entornos de alta competitividad.

Asimismo, esta revisión presenta espacios de interés para futuras investigaciones, tanto dentro de las capacidades de los socios participantes en una alianza como de las particularidades del conocimiento transmitido (Meier, 2011). Además, se espera haber aclarado el campo de las capacidades de aprendizaje y la construcción de ventajas competitivas con fines prácticos, así como fomentar el acercamiento de ejecutivos y académicos que deseen hacer su primera aproximación a una literatura que aún tiene mucho por ofrecer. 


\section{bibliografía}

Adler, P. S. \& S. Kwon

2002

Prospects for a new concept. Academy

of Management Review, 27(1), 17-40.

Recuperado de: <https://doi.org/10.5465/

AMR.2002.5922314>

\section{Arora, A. \& A. Gambardella}

2010

Ideas for rent: An overview of markets

for technology. Industrial and Corporate

Change, 19(3), 775-803. Recuperado de:

$<$ https://doi.org/10.1093/icc/dtq022>.

\section{Augier, M. \& D. J. Teece}

2009 Dynamic capabilities and the role of managers in business strategy and economic performance. Organization Science, 20(2), 410-421. Recuperado de: $<$ https://doi.org/10.1287/orsc. 1090.0424>.

\section{Barney, J.}

Firms resources and sustained competitive advantage. Journal of Management, 17(45), 99-120. Recuperado de: <https:// doi.org/10.1177/014920639101700108>.

\section{Barreto, I.}

2010 Dynamic capabilities: A review of past research and an agenda for the future. Journal of Management, 36, 256-280. Recuperado de: <https://doi. org/10.1177/0149206309350776>.

\section{Beamish, P. W. \& N. C. Lupton}

Managing joint ventures. Academy of Management Perspectives, 23(2), 75-94. Recuperado de: <https://doi.org/10.5465/ AMP.2009.39985542>.

\section{Birkinshaw, J.; R. Nobel \&}

\section{J. Ridderstrale}

Knowledge as a contingency variable: predict organization structure?

Organization Science, 13(3), 274-289.

Recuperado de: <https://doi.org/10.1287/

orsc. 13.3.274.2778>

\section{Cassiman, B. \& R. Veugelers}

2006

In search of complementarity in innovation strategy: Internal R\&D and external knowledge acquisition. Management Science, 52(1), 68-82. Recuperado de: $<$ https://doi.org/10.1287/mnsc.1050.0470>

\section{Cepeda, G. \& D. Vera}

2007 Dynamic capabilities and operational capabilities: A knowledge management perspective. Journal of Business Research, 60(5), 426-437. Recuperado de: <https:// doi.org/10.1016/i.jbusres.2007.01.013>

\section{Chen, C. J.;Y. C. Hsiao \&}

\section{A. Chu}

2014 Transfer mechanisms and knowledge transfer: The cooperative competency perspective. Journal of Business Research, 67(12), 2531-2541. Recuperado de: <https://doi.org/10.1016/j. jbusres.2014.03.011>

\section{Chen, R.; S. Gallagher \&}

\section{S. H. Park}

Firm resources as moderators of the

$$
\text { relationship between market growth }
$$
and strategic alliances in semiconductor start-ups. The Academy of Management Journal, 45(3), 527-545.

\section{Chesbrough, H.; W.}

\section{Vanhaverbeke \& J. West}

2006

Open Innovation: Researching a New

Paradigm. Oxford: Oxford University Press.

Do the characteristics of knowledge 


\section{Christoffersen, J.;T. Plenborg \&} M. J. Robson

2014

Measures of strategic alliance

performance, classified and assessed.

International Business Review, 23(3),

479-489. Recuperado de: <https://doi.

org/10.1016/j.ibusrev.2013.07.001>.

\section{Coase, R. H.}

The nature of the firm. Economica, 4(16), 386-405. Recuperado de: <https://doi. org/10.2307/2626876>.

\section{Cohen, W. \& D. Levinthal}

1990

Absorptive capacity: A new

perspective on learning and innovation.

Administrative Science Quarterly, 35(1),

128-52. Recuperado de: <https://doi.

org/10.2307/2393553>

\section{Cropper, S.; M. Ebers, C.}

\section{Huxham \& P. S. Ring}

Introducing inter-organizational relations.

The Oxford Handbook of Inter-

Organizational Relations, (mayo), 1-15.

Recuperado de: <https://doi.org/10.1093/

oxfordhb/9780199282944.003.0001>

\section{Crossan, M. M. \& M. Apaydin}

2010

A multi-dimensional framework of

organizational innovation: A systematic

review of the literature. Journal of

Management Studies, 47(6), 1154-1191.

Recuperado de: <https://doi.org/10.1111

|j.1467-6486.2009.00880.x>.

\section{Das, T. K. \& B. S. Teng}

1998

Between trust and control: Developing

confidence in partner cooperation in

alliances. Academy of Management

Review, 23(3), 491-512. Recuperado

de: <https://doi.org/10.5465/

AMR.1998.926623>.

\section{Das, T. K. \& B. S. Teng}

2000

A resource-based theory of strategic

alliances. Journal of Management, 26(1),

31-61. Recuperado de: <https://doi.

org/10.1016/S0149-2063(99)00037-9>.

\section{Dhanaraj, C.; M. A. Lyles,}

\section{H. K. Steensma \& L. Tihanyi}

2004

Managing tacit and explicit knowledge

transfer in IJVs: The role of relational

embeddedness and the impact on

performance. Journal of International

Business Studies, 35(5), 428-442.

Recuperado de: <https://doi.org/10.1057/

palgrave.jibs.8400098>

\section{Díaz Gamarra, M. A.}

2017

Efectos de la transferencia de conocimiento en las alianzas para la innovación. Tesis de Maestría. Universidad ESAN, Perú.

\section{Dyer, J. H.; P. Kale \& H. Singh}

How to make strategic alliances work. MIT Sloan Management Review, 42(4), 37.

\section{Fagerberg, J.}

2004

Innovation: A guide to the literature. En J. Fagerberg, D. C. Mowery y R. R. Nelson (eds.), The Oxford Handbook of Innovation (pp. 1-26). Oxford: Oxford University Press.

\section{Faulkner, D. 0.}

Cooperative strategy: Strategic alliances and networks. En The Oxford Handbook of Strategy: A Strategy Overview and Competitive Strategy (pp. 610-648).

Oxford: Oxford University Press. 


\section{bibliografía}

Recuperado de: <https://doi.org/10.1093/

oxfordhb/9780199275212.003.0021>.

\section{Frost, T. S.; J. M. Birkinshaw \& P. C. Ensign}

2002

Centers of excellence in multinational corporations. Strategic Management Journal, 23(11), 997-1018. Recuperado de: $<\underline{\text { https://doi.org/10.1002/smj.273> }}$

\section{Gassmann, 0 .}

2006 Opening up the innovation process: Towards an agenda. $R$ and D Management, 36(3), 223-228. Recuperado de: <https:// doi.org/10.1111/i.1467-9310.2006.00437.x>

Geyskens, I.; J. B. E. M.

\section{Steenkamp \& N. Kumar}

Make, buy, or ally: A transaction cost

theory meta-analysis. Academy of

Management Journal, 49(3), 519-543.

Recuperado de: <https://doi.org/10.5465/

AMJ.2006.21794670>.

\section{Globerman, S. \& B. B. Nielsen}

2007 Equity versus non-equity international strategic alliances involving Danish firms: An empirical investigation of the relative importance of partner and host country determinants. Journal of International Management, 13(4), 449-471. Recuperado de: <https://doi.org/10.1016/j. intman.2007.03.005>.

\section{Gomes, E.; B. R. Barnes \&}

\section{T. Mahmood}

A 22 year review of strategic alliance research in the leading management journals. International Business Review, 25(1), 15-27 Recuperado de: <https://doi. org/10.1016/i.ibusrev.2014.03.005> .

\section{Grant, R. M.}

1996

Toward a knowledge-based theory of the firm. Strategic Management Journal, 17(S2), 109-122. Recuperado de: <https:// doi.org/10.1002/smi.4250171110>.

\section{Grant, R. M.}

2006 The knowledge-based view of the firm. En A. Campbell y D. O. Faulkner (eds.), The Oxford Handbook of Strategy: A Strategy Overview and Competitive Strategy (pp. 203-230). Recuperado de: <https://doi.org/10.1093/ oxfordhb/9780199275212.003.0008>.

\section{Grant, R. M. \& C. Baden-Fuller}

2004 A knowledge accessing theory of strategic alliances. Journal of Management Studies, 41(1), 61-84. Recuperado de: <https://doi. org/10.1111/i.1467-6486.2004.00421.x>.

\section{Gray, P. H. \& D. B. Meister}

2004 Knowledge sourcing effectiveness. Management Science, 50(6), 821-834. Recuperado de: <https://doi.org/10.1287/ mnsc. 1030.0192>.

\section{Gulati, R.}

1998 Alliances and networks. Strategic Management Journal, 19, 293-317. Recuperado de: $<$ https://doi. org/10.2307/3094067>

\section{Gulati, R. \& H. Singh}

The architecture of cooperation: Managing coordination costs and appropriation concerns in strategic alliances. Administrative Science Quarterly, 43(4), 781-814. Recuperado de: <https://doi. org/10.2307/2393616>. 


\section{bibliografía}

Gunday, G.; G. Ulusoy, K. Kilic

\section{\& L. Alpkan}

2011

Effects of innovation types on firm

performance. International Journal of

Production Economics, 133(2), 662-676.

Recuperado de: <https://doi.org/10.1016/j.

ijpe.2011.05.014>.

\section{Gupta, A. K. \& V. Govindarajan}

2000

Knowledge flows within multinational

corporations. Strategic Management

Journal, 21(4), 473-496.

\section{Helfat, C. E. \& M. A. Peteraf}

2003

Capability lifecycles. Strategic

Management Journal, 24, 997-1010.

Recuperado de: <https://doi.org/10.1002/ smi.332>.

\section{Inkpen, A. C. \& A. Dinur}

1998

Knowledge management processes and international joint ventures. Organization Science, 9(4), 454-468. Recuperado de: $<$ https://doi.org/10.1287/orsc.9.4.454>

\section{Inkpen, A. C. \& E. W. K. Tsang}

Learning and strategic alliances.

Academy of Management Annals, 1(1) 479-511. Recuperado de: <https://doi. org/10.1080/078559815>

\section{Jiménez-Jiménez, D. \&}

\section{R. Sanz-Valle}

and performance. Journal of Business

Research, 64(4), 408-417. Recuperado

de: <https://doi.org/10.1016/i.

jbusres.2010.09.010>
Johnson, R. A.; F. E. Kast \&

\section{J. E. Rosenzweig}

1964

Systems theory and management.

Management Science, 10(2),

367-384. Recuperado de: <https://doi. org/10.2307/2627306>

\section{Kale, P. \& H. Singh}

2007

Building firm capabilities through learning:

The role of the alliance learning process

in alliance capability and firm-level alliance success. Strategic Management Journal, 28(10), 981-1000. Recuperado de: <https:// doi.org/10.1002/smj.616>.

\section{Khanna,T.; R. Gulati \& \\ N. Nohria}

1998

The dynamics of learning alliances:

Competition, cooperation, and relative scope. Strategic Management Journal, 19(3), 193-210. Recuperado de: $<$ https://doi.org/10.1002/(SICI)10970266(199803)19:3<193::AID-

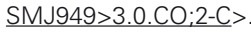

\section{Kogut, B.}

Joint ventures: Theoretical and empirical perspectives. Strategic Management Journal, 9, 319-332. Recuperado de: <https://doi.org/10.1002/smj.4250090403>.

\section{Kogut, B. \& U. Zander}

1992

Knowledge of the firm, combinative capabilities, and the replication of technology. Organization Science, 3(3), 383-397. Retrieved from Recuperado de: $<$ http://www.jstor.org/stable/2635279>. 


\section{bibliografía}

Koza, M. P. \& A. Y. Lewin

1998

The co-evolution of strategic alliances.

Organization Science, 9(3), 255-264.

Recuperado de: <https://doi.org/10.1287/

orsc.9.3.255>.

\section{Lane, P. J. \& M. Lubatkin}

1998 Relative absorptive capacity and interorganizational learning. Strategic Management Journal, 19(noviembre), 461-477.

\section{Laursen, K. \& A. Salter}

2006

Open for innovation: The role of openness

in explaining innovation performance

among U.K. manufacturing firms. Strategic

Management Journal, 27(2), 131-150.

Recuperado de: <https://doi.org/10.1002/ smj.507>

\section{Levine, S. \& M. Prietula}

How knowledge transfer impacts

performance: A multilevel model of

benefits and liabilities. Organization

Science, 23(6), 1448-1766. Recuperado de:

$<$ https://doi.org/10.1287/orsc. 1110.0697>.

\section{Lichtenthaler, U.}

2011 Open innovation: Past research, current debates, and future directions. Academy of Management Perspectives, 25(1), 75-93. Recuperado de: <https://doi.org/10.5465/ AMP.2011.59198451>.

\section{Lin, C.; Y. J. Wu, C. Chang, W. Wang \& C.Y. Lee}

The alliance innovation performance of $R \& D$ alliances - The absorptive capacity perspective. Technovation, 32(5), 282-292. Recuperado de: <https://doi.org/10.1016/j. technovation.2012.01.004>
March, J. G.

1991 Exploration and exploitation in organizational learning. Organization Science, 2(1), 71-87.

\section{Marshall, R. S.; T. V. Nguyen \&}

\section{S. E. Bryant}

2005

A dynamic model of trust development and knowledge sharing in strategic alliances. Journal of General Management, 31(1), 41-57.

\section{Meier, $\mathbf{M}$.}

2011 Knowledge management in strategic alliances: A review of empirical evidence. International Journal of Management Reviews, 13(1), 1-23.

Recuperado de: <https://doi.org/10.1111 〈j.1468-2370.2010.00287.x>.

\section{Mowery, D. C.; J. E. Oxley \&}

\section{B. S. Silverman}

1996 Strategic alliance and interfirm knowledge transfer. Strategic Management Journal, 17(Winter Special Issue), 77-91. Recuperado de: <https://doi. org/10.1055/s-0031-1275710>

\section{Nonaka, I.}

1994 A dynamic theory of organizational knowledge creation. Organization Science, 5(1), 14-37. Recuperado de: <https://doi. org/10.1287/orsc.5.1.14>

\section{Nonaka, I. \& G. von Krogh}

Perspective-tacit knowledge and knowledge conversion: Controversy and advancement in organizational knowledge creation theory. Organization Science, 20(3), 635-652. Recuperado de: <https:// doi.org/10.1287/orsc. 1080.0412>. 


\section{bibliografía}

Nooteboom, B.; W. Van

Haverbeke, G. Duysters, V.

Gilsing \& A. van den Oord

2007

Optimal cognitive distance and absorptive capacity. Research Policy, 36(7), 1016-1034.

Recuperado de: <https://doi.org/10.1016/j. respol.2007.04.003>.

\section{Noseleit, F. \& P. de Faria}

2013

Complementarities of internal $R \& D$ and alliances with different partner types. Journal of Business Research, 66(10), 2000-2006. Recuperado de: <https://doi. org/10.1016/i.jbusres.2013.02.025> .

\section{Ortiz-de-Urbina-Criado, M.;}

Á. Montoro-Sánchez \&

\section{E. M. Mora-Valentín}

Impact of growth strategy on mode of governance in alliances. International Business Review, 23(4), 838-848. Recuperado de: <https://doi.org/10.1016/j. ibusrev.2014.01.002>.

Oxley, J. E.

Appropriability hazards and governance in strategic alliances: A transaction cost approach. Journal of Law, Economics \& Organization, 13(2), 387-409.

\section{Park, S. H. \& G. R. Ungson}

Interfirm rivalry and managerial complexity : A conceptual framework of alliance failure. Organization Science, 12(1), 37-53. Recuperado de: <https://doi. org/10.1287/orsc.12.1.37.10118>.

\section{Parmigiani, A. \&}

\section{Rivera-Santos}

Journal of Management, 37(4), 1108-1136. Recuperado de: <https://doi. org/10.1177/0149206311407507>.

\section{Pavitt, K.}

2009

Innovation processes. The Oxford

Handbook of Innovation, (mayo), 1-21.

Recuperado de: <https://doi.org/10.1093/ oxfordhb/9780199286805.003.0004>

\section{Peteraf, M. A.}

1993

The cornerstones of competitive advantage: A resource-based view. Strategic Management Journal, 14(3), 179-191. Recuperado de: <https://doi. org/10.1002/smj.4250140303> .

\section{Pfeffer, J. \& G. Salancik}

1978

Social control of organizations. En The External Control of Organizations: A Resource Dependence Perspective (pp. 39-52). Stanford: Stanford Business Books. Recuperado de: <https://doi. org/10.2307/2392573>

\section{Pittaway, L.; M. Robertson, K. Munir, D. Denyer \& A. Neely}

Networking and innovation: A systematic review of the evidence. International Journal of Management Reviews, 5-6(3-4), 137-168. Recuperado de: <https://doi.org/1

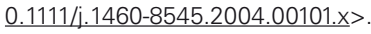

\section{Polanyi, M.}

The logic of tacit inference. Philosophy, XL/(155), 1-18. Recuperado de: <https://doi. org/10.1017/S0031819100066110>.

\section{Polanyi, M. \& A. Sen}

The Tacit Dimension. Chicago: University of Chicago Press. 


\section{bibliografía}

\section{Provan, K. G. \& J. Sydow}

2009

Evaluating inter-organizational

relationships. En The Oxford Handbook of Inter-Organizational Relations (pp. 1-18).

Recuperado de: <https://doi.org/10.1093/ oxfordhb/9780199282944.003.0026>

\section{Sambasivan, M.; L. Siew-Phaik,}

\section{Z. Abidin Mohamed \&}

\section{Y. C. Leong}

Factors influencing strategic alliance

outcomes in a manufacturing supply

chain: Role of alliance motives,

interdependence, asset specificity and

relational capital. International Journal of

Production Economics, 141(1), 339-351.

Recuperado de: <https://doi.org/10.1016/j.

ipe.2012.08.016>.

\section{Sampson, R. C.}

$R \& D$ alliances and firm performance:

The impact of technological diversity and alliance organization on innovation. Academy of Management Journal, 50(2), 364-386. Recuperado de: <https://doi. org/10.5465/AMJ.2007.24634443>.

\section{Sheremata, W. A.}

Centrifugal and centripetal forces in radical new product development under time pressure. Academy of Management Review, 25(2), 389-408.

\section{Simonin, B. L.}

Ambiguity and the process of knowledge transfer in strategic alliances. Strategic Management Journal, 20(7), 595-623. Recuperado de: <https://doi.org/10.1002/ (SICI)1097-0266(199907)20:7<595::AIDSMJ47>3.0.CO;2-5>
Simonin, B. L.

2004 An empirical investigation of the process of knowledge transfer in international strategic alliances. Journal of International Business Studies, 35(5), 407-427. Recuperado de: <https://doi.org/10.1057/ palgrave.jibs.8400091>

\section{Smith, K. G.; S. J. Carroll \&}

\section{S. J. Ashford}

Intra- and interorganizational cooperation: Toward a research agenda. Academy of Management Journal, 38(1), 7-23. Recuperado de: <https://doi. org/10.2307/256726 >

Smith, K. G.; C. J. Collins \&

\section{K. D. Clark}

2005 Existing knowledge, knowledge creation capability, and the rate of new product introduction in high-technology firms. Academy of Management Journal, 48(2), 346-357. Recuperado de: <https://doi. org/10.5465/AMJ.2005.16928421>.

\section{Spender, J. C. \& R. M. Grant}

1996 Knowledge and the firm: Overview. Strategic Management Journal, 17(2), 5-9. Recuperado de: <https://doi.org/10.1002/ smj.4250171103>.

\section{Su, Z.; D. Ahlstrom, J. Li \& \\ D. Cheng}

Knowledge creation capability, absorptive capacity, and product innovativeness. $R$ and D Management, 43(5), 473-485. Recuperado de: <https://doi.org/10.1111/ $\underline{\operatorname{radm} .12033>}$ 


\section{bibliografía}

Szulanski, G.

2003

Sticky Knowledge: Barriers to Knowing in

the Firm. Thousand Oaks, California: Sage Publications.

\section{Szulanski, G.; D. Ringov \&}

\section{R. J. Jensen}

2016

Overcoming stickiness: How the timing

of knowledge transfer methods affects

transfer difficulty. Organization Science,

27(2), 304-322. Recuperado de: <https://

doi.org/10.1287/orsc.2016.1049>.

\section{Tallman, S. B.}

2006

Dynamic capabilities. En A. Campbell y D.

O. Faulkner (eds.), The Oxford Handbook

of Strategy: A Strategy Overview

and Competitive Strategy (XVIII, pp.

1-29). Oxford: Oxford University Press.

Recuperado de: <https://doi.org/10.1093/

oxfordhb/9780199275212.003.0013>

Teece, D. J.

2010

Forward integration and innovation:

Transaction costs and beyond.

Journal of Retailing, 86(3), 277-283.

Recuperado de: <https://doi.org/10.1016/j.

iretai.2010.07.013>.

\section{Teece, D. J.; G. Pisano \&}

\section{A. Shuen}

1997

Dynamic capabilities and strategic

management. Strategic Management

Journal, 18(7), 509-533. Recuperado

de: <https://doi.org/10.1002/

(SICI)1097-0266(199708)18:7<509::AID-

SMJ882>3.0.CO:2-Z> .

\section{Todeva, E. \& D. Knoke}

Strategic alliances and models of

collaboration. Management Decision,
43(1), 123-148. Recuperado de: <https:// doi.org/10.1108/00251740510572533>.

\section{Tsai, W.}

2001

Knowledge transfer in intraorganizational networks: Effects of network position and absorptive capacity on business unit innovation and performance. Academy of Management Journal, 44(5), 996-1004.

\section{Tsoukas, H. \& E. Vladimirou}

2001

What is organizational knowledge? Journal of Management Studies, 38(7), 973-993. Recuperado de: <https://doi. org/10.1111/1467-6486.00268>

\section{Van De Vrande, V.; C. Lemmens \& W. Vanhaverbeke}

Choosing governance modes for external technology sourcing. $R$ and D Management, 36(3), 347-363. Recuperado de: <https://doi.org/10.1111 Li.1467-9310.2006.00434.x>

\section{Vanhaverbeke, W.; V. Van De Vrande \& H.W. Chesbrough}

Understanding the advantages of open innovation practices incorporate venturing in term of real options. Creativity and Innovation Management, 17(4), 251-258. Recuperado de: <https://doi.org/10.1111 Li.1467-8691.2008.00499.x>.

\section{Van Wijk, R.; J. J. P. Jansen \&} M. A. Lyles

Inter- and intra-organizational knowledge transfer: A meta-analytic review and assessment of its antecedents and consequences. Journal of Management Studies, 45(4), 830-853. Recuperado de: <https://doi.org/10.1111 〈j.1467-6486.2008.00771.x>. 


\section{bibliografía}

Vera, D.; M. Crossan \& M.

Apaydin

2011

A framework for integrating organizational learning, knowledge, capabilities, and absorptive capacity. En Handbook

of Organizational Learning and Knowledge Management (pp. 153-180) Hoboken, Nueva Jersey: John Wiley \& Sons. Recuperado de: <https://doi. org/10.1002/9781119207245.ch8>

\section{Wang, Y. H. \& D. J. Miao}

Using strategic alliances to make decisions about investing in technological innovations. International Journal of Management, 23(1), 195-200. Recuperado de: <http://search.ebscohost.com/login.asp $\underline{x}$ ?direct $=$ true $\& d b=$ buh $\& A N=20302807 \&$ si te $=$ ehost-live $>$.
Williamson, O. E.

2010

Transaction cost economics: The natural progression. Journal of Retailing, 86(3), 215-226. Recuperado de: <https://doi. org/10.1016/i.jretai.2010.07.005>

Young-Ybarra, C. \& M.

\section{Wiersema}

1999

Strategic flexibility in information

technology alliances: The influence of

transaction cost economics and social

exchange theory strategic flexibility

in information technology alliances.

Organization Science, 10(4), 439-459.

Recuperado de: <https://doi.org/10.1287/

orsc. $10.4 .439>$

Fecha de recepción: 07/04/2017

Fecha de aceptación: 01/06/2017 